\title{
Terapias de base cognitivo-comportamental do transtorno obsessivo-compulsivo: revisão sistemática da última década
} Cognitive-behavior therapy for obsessive-compulsive
disorder: a systematic review of the last decade

\author{
Angélica Marques Prazeres, ${ }^{1}$ Wanderson Fernandes de Souza, ${ }^{1}$ \\ Leonardo F Fontenelle ${ }^{1}$
}

\begin{abstract}
Resumo
Objetivo: Revisar de forma sistemática os estudos controlados e meta-análises que envolveram tratamento cognitivo ou comportamental do transtorno obsessivo-compulsivo publicados na última década. Método: Análise sistemática de ensaios clínicos randomizados e meta-análises indexados no Medline e Psyclnfo. Resultados: Os estudos avaliados confirmam que a exposição e prevenção de resposta e a terapia cognitiva são eficazes no tratamento do transtorno obsessivo-compulsivo de crianças, adolescentes e adultos. Em adultos, o uso concomitante de inibidores da recaptação da serotonina e exposição e prevenção de resposta ou terapia cognitiva não foi associado a um efeito adicional na resposta ao tratamento. No transtorno obsessivo-compulsivo infantil, o uso combinado de inibidores da recaptação da serotonina e exposição e prevenção de resposta foi superior aos dois tratamentos isoladamente. A exposição e prevenção de resposta associada à terapia cognitiva resultou em benefícios significativos em pacientes com predominância de pensamentos obsessivos e sua modalidade em grupo também produziu redução significativa dos sintomas obsessivos e compulsivos. Conclusão: Atualmente, as terapias de base cognitivocomportamental são as mais adequadas para o tratamento do transtorno obsessivo-compulsivo; porém, mais estudos envolvendo follow-up em longo prazo, tratamento em grupo e uso concomitante de medicação são necessários.
\end{abstract}

Descritores: Transtorno obsessivo-compulsivo; Terapia comportamental; Terapia cognitiva; Ensaios clínicos controlados; Literatura de revisão

\begin{abstract}
Objective: To perform a systematic review of controlled trials and meta-analysis that involved cognitive and/or behavioral treatment for obsessive-compulsive disorder. Method: A systematic review of randomized controlled trials and meta-analysis published on the last decade and indexed on Medline and Psyclnfo. Results: Studies have confirmed that exposure and response prevention and cognitive therapy are effective methods for the treatment of obsessive-compulsive disorder in children, adolescents and adults. Among adults, the combined use of serotonin-reuptake inhibitors and exposure and response prevention or cognitive therapy was not associated with any additional therapeutic effect. Among children, the combination of serotonin-reuptake inhibitors and exposure and response prevention are superior to either treatment alone. Exposure and response prevention associated with cognitive therapy may result in significant benefits to patients with predominant obsessive thoughts and its group modality also reduces obsessive-compulsive symptoms. Conclusion: At the present time, cognitive and behavioral therapies have shown to be highly effective psychotherapeutic approaches for the treatment of obsessive-compulsive disorder. Nevertheless, more studies are still needed, mainly those focusing on long-term follow-up, group-treatment and the combined use with serotonin-reuptake inhibitors.
\end{abstract}

Descriptors: Obsessive-compulsive disorder; Behavior therapy; Cognitive therapy; Randomized controlled trials; Review literature

1 Programa de Pesquisa em Ansiedade e Depressão, Instituto de Psiquiatria, Universidade Federal do Rio de Janeiro (IPUB/UFRJ), Rio de Janeiro (RJ), Brasil

Financiamento: Inexistente

Conflito de interesses: Inexistente

Submetido: 10 Outubro 2006

Aceito: 26 Fevereiro 2007
Correspondência

Leonardo F. Fontenelle

Rua Otávio Carneiro, 93 - apto. 601 - Icaraí

24230-190 Niterói, RJ, Brasil

E-mail: Ifontenelle@gmail.com 


\section{Introdução}

O transtorno obsessivo-compulsivo (TOC) é um transtorno psiquiátrico comum, com prevalência durante a vida estimada entre $0,3 \%$ e $2,2 \%$ em adultos e aproximadamente $0,7 \%$ em crianças e adolescentes, de acordo com dados baseados no Composite International Diagnostic Interview (CIDI). ${ }^{1} \mathrm{O}$ TOC caracteriza-se pela presença de pensamentos, idéias ou imagens intrusivos e involuntários que provocam ansiedade (obsessões) e por rituais mentais ou comportamentais realizados para neutralizar a ansiedade (compulsões). ${ }^{2}$

Em um estudo realizado com 70 pacientes com diferentes tipos de transtornos de ansiedade, observou-se que pacientes com TOC têm menor probabilidade de remissão espontânea dos sintomas se comparados a pacientes com transtornos de pânico e de ansiedade social. ${ }^{3}$ Este resultado demonstra a necessidade do desenvolvimento e aprimoramento dos tratamentos para o TOC. As terapias de base cognitivocomportamental, e.g. exposição e prevenção de respostas (EPR) e terapia cognitiva (TCog), representam as alternativas psicoterápicas mais estudadas até o momento. ${ }^{4}$

A EPR baseia-se na relação funcional entre obsessões e compulsões e objetiva enfraquecer as associações entre obsessões e aumento da ansiedade e entre compulsões e alívio de ansiedade. ${ }^{2}$ Para tal, envolve repetidas e prolongadas confrontações com o estímulo que dispara o medo obsessivo e, simultaneamente, o impedimento ou a interrupção dos comportamentos compulsivos associados. ${ }^{5}$ Quando a obsessão não é seguida por rituais, o paciente aprende que as compulsões não são necessárias para prevenir desastres ou reduzir a ansiedade. ${ }^{2}$ Mesmo sendo considerada um tratamento efetivo para o TOC, há pacientes que não aderem à EPR, principalmente aqueles que apresentam obsessões puras ou cujas crenças são supervalorizadas. ${ }^{6}$

Já a TCog para o tratamento do TOC é baseada no modelo de terapia cognitiva para transtornos depressivos e ansiosos de Beck..$^{7-9}$ As estratégias mais utilizadas são a eliciação e a discussão dos pensamentos automáticos e intrusivos, do medo disfuncional e dos esquemas de responsabilidade; a modificação de interpretações irrealistas; e a discussão sobre pensamento mágico e fusão de pensamento e ação. O esclarecimento sobre os efeitos de não ritualizar e o questionamento socrático são importantes técnicas cognitivas usadas pelos terapeutas. Além disso, experimentos comportamentais são usados como teste para os pensamentos automáticos distorcidos. ${ }^{7-10}$ Alguns estudos têm proposto a utilização da TCog aliada à EPR com o objetivo de aumentar a adesão e a resposta ao tratamento de pacientes com TOC.

Desde a década de 70, diversos estudos mostraram que terapias de base cognitivo-comportamental são tratamentos eficazes que produzem uma melhora em $60-85 \%$ dos pacientes com TOC. ${ }^{11}$ Em 1997, Isaac Marks conduziu uma importante revisão dos principais artigos sobre terapias de base cognitivocomportamental no tratamento do TOC publicados na década anterior. ${ }^{5} \mathrm{Na}$ ocasião, destacaram-se estudos que demonstravam a eficácia da EPR no tratamento de pacientes com TOC de todos os grupos etários; a persistência dos ganhos terapêuticos em estudos de follow-up; a melhora do humor, do funcionamento ocupacional e do ajustamento social; a necessidade de medicação antidepressiva em pacientes com depressão comórbida em tratamento com EPR; o valor da auto-exposição; e, finalmente, uma possível equivalência terapêutica entre a EPR e a TCog.

Neste estudo, o nosso objetivo foi revisar de forma sistemática as pesquisas controladas que envolveram terapia cognitiva ou comportamental para o tratamento do TOC realizadas na última década. Pretendemos, portanto, avaliar o progresso do tratamento psicológico do TOC dez anos após a importante revisão de Marks. ${ }^{5}$

\section{Método}

Os estudos de tratamento do TOC foram identificados através de busca nos bancos de dados do PubMed/Medline e Psyclnfo em dezembro de 2006, utilizando as seguintes expressões: (CBT OR cognitive behaviour therapy OR behaviour therapy OR cognitive therapy) AND (OCD OR obsessive compulsive disorder OR compulsive disorder OR obsessive disorder). A busca limitou-se aos ensaios clínicos randomizados e meta-análises publicados entre 1995 e 2006, utilizando-se os filtros existentes nos bancos de dados. Somente estudos em inglês, alemão, francês, espanhol e português foram avaliados. Apenas ensaios clínicos randomizados realizados com pacientes com diagnóstico primário de TOC tratados com psicoterapia de abordagem cognitiva ou comportamental e meta-análises que avaliaram a eficácia de tratamentos desta mesma abordagem foram incluídos.

Inicialmente, por meio do nosso critério de busca na base de dados do PubMed/Medline, 63 artigos controlados e 5 metaanálises foram obtidos, dos quais 32 estudos controlados e 3 meta-análises preencheram os critérios de inclusão. No Psyclnfo, a busca resultou em 38 artigos controlados e 9 metaanálises, dos quais 7 estudos controlados (sendo 6 artigos comuns ao PubMed/Medline) e 6 meta-análises (sendo um comum ao PubMed/Medline) preencheram os critérios de inclusão. Todos os artigos foram inspecionados e avaliados por um psicólogo e uma aluna de psicologia do último ano. Após a análise, um total de 33 estudos controlados e 8 meta-análises foram incluídos na revisão (Tabela 1 e Tabela 2). Os artigos obtidos que não foram incluídos não se referiam a estudos controlados ou a meta-análises, não incluíam pacientes com TOC ou não focavam em terapia cognitiva ou comportamental.

\section{Resultados}

Os ensaios clínicos controlados localizados em nossa busca encontram-se listados na Tabela 1 e as meta-análises na Tabela 2. Uma descrição mais detalhada de cada tipo de estudo é fornecida logo abaixo.

\section{EPR vs. relaxamento}

Em um estudo controlado, 18 pacientes com TOC foram divididos em dois grupos de tratamento: EPR ou técnicas de controle de ansiedade (grupo controle). O grupo que recebeu tratamento com EPR obteve redução estatisticamente significativa dos sintomas obsessivo-compulsivos, mas nenhuma alteração em relação à condição inicial do pré-tratamento foi observada no grupo controle. ${ }^{12}$

\section{EPR vs. exposição imaginária (ExI)}

Dois estudos avaliaram o quanto a adição de exposição imaginária à exposição ao vivo e prevenção de resposta (EPR) poderia aumentar os ganhos no TOC. Quarenta e seis pacientes com TOC foram submetidos às sessões de EPR ou às sessões de exposições imaginárias sobre as conseqüências de não ritualizar (ExI) associadas às sessões de EPR. A adição de ExI à exposição ao vivo não representou melhora em relação ao grupo que recebeu apenas a exposição ao vivo. ${ }^{13-14}$ 
Tabela 1 - Estudos controlados realizados entre 1995 e 2006

\begin{tabular}{|c|c|c|c|}
\hline Estudo & Tratamento & $\mathbf{n}$ & $\begin{array}{l}\text { Duração do } \\
\text { tratamento }\end{array}$ \\
\hline Van Oppen 1995 & $\begin{array}{l}\text { EPR } \\
\text { TCog }\end{array}$ & $\begin{array}{l}29 \\
28\end{array}$ & 16 semanas \\
\hline $\begin{array}{l}\text { Araujo 1995, lto } \\
1995, \text { Araujo } 1996\end{array}$ & $\begin{array}{l}\text { EPR } \\
\text { EPR+Exi }\end{array}$ & $\begin{array}{l}23 \\
23\end{array}$ & 9 semanas \\
\hline Lindsay 1997 & $\begin{array}{l}\text { EPR } \\
\text { Relaxamento }\end{array}$ & $\begin{array}{l}9 \\
9\end{array}$ & 3 semanas \\
\hline Freeston 1997 & $\begin{array}{l}\text { EPR+TCog } \\
\text { lista de espera }\end{array}$ & $\begin{array}{l}15 \\
14\end{array}$ & $\begin{array}{l}25,7 \text { semanas } \\
\text { (média) }\end{array}$ \\
\hline de Haan 1997 & $\begin{array}{l}\text { EPR } \\
\text { TCog } \\
\text { EPR+Fluvoxamina } \\
\text { TCog+Fluvoxamina }\end{array}$ & $\begin{array}{l}22 \\
25 \\
28 \\
24\end{array}$ & 16 semanas \\
\hline Dreessen 1997 & $\begin{array}{l}\text { EPR } \\
\text { TCog } \\
\text { EPR+TCog }\end{array}$ & $\begin{array}{l}16 \\
14 \\
22\end{array}$ & 12 semanas \\
\hline Hohagen 1998 & $\begin{array}{l}\text { EPR+Fluvoxamina } \\
\text { EPR+placebo }\end{array}$ & $\begin{array}{l}30 \\
30\end{array}$ & 9 semanas \\
\hline Van Balkom 1998 & $\begin{array}{l}\text { EPR } \\
\text { TCog } \\
\text { EPR+Fluvoxamina } \\
\text { TCog+Fluvoxamina } \\
\text { lista de espera }\end{array}$ & $\begin{array}{l}22 \\
25 \\
28 \\
24 \\
18\end{array}$ & 16 semanas \\
\hline Neziroglu 2000 & $\begin{array}{l}\text { Fluvoxamina } \\
\text { Fluvoxamina+EPR }\end{array}$ & $\begin{array}{l}5 \\
5\end{array}$ & $\begin{array}{l}10 \text { semanas de } \\
\text { FVM } \\
\text { +20 semanas de } \\
\text { EPR }\end{array}$ \\
\hline McLean 2001 & $\begin{array}{l}\text { TCog grupo } \\
\text { EPR grupo }\end{array}$ & $\begin{array}{l}34 \\
42\end{array}$ & 12 semanas \\
\hline Cottraux 2001 & $\begin{array}{l}\text { EPR } \\
\text { TCog }\end{array}$ & $\begin{array}{l}33 \\
32\end{array}$ & 16 semanas \\
\hline $\begin{array}{l}\text { Greist 2002, Mataix } \\
2002\end{array}$ & $\begin{array}{l}\text { EPR computador } \\
\text { EPR terapeuta } \\
\text { Relaxamento } \\
\text { sistemático }\end{array}$ & $\begin{array}{l}74 \\
69 \\
75\end{array}$ & 10 semanas \\
\hline Volpato 2003 & $\begin{array}{l}\text { TCog grupo } \\
\text { lista de espera }\end{array}$ & $\begin{array}{l}23 \\
24\end{array}$ & 12 semanas \\
\hline Barrett 2004 & $\begin{array}{l}\text { EPR+TCog Familiar } \\
\text { grupo } \\
\text { EPR+TCog Familiar } \\
\text { individual } \\
\text { lista de espera }\end{array}$ & $\begin{array}{l}29 \\
24 \\
24\end{array}$ & 14 semanas \\
\hline POTS 2004 & $\begin{array}{l}\text { EPR+TCog } \\
\text { Sertralina } \\
\text { EPR+TCog }+ \\
\text { Sertralina } \\
\text { Placebo }\end{array}$ & $\begin{array}{l}28 \\
28\end{array}$ & 12 semanas \\
\hline $\begin{array}{l}\text { Simpson 2004, Foa } \\
2005\end{array}$ & $\begin{array}{l}\text { EPR } \\
\text { Clomipramina } \\
\text { EPR+Clomipramina } \\
\text { Placebo }\end{array}$ & $\begin{array}{l}29 \\
36 \\
31 \\
26\end{array}$ & 12 semanas \\
\hline $\begin{array}{l}\text { Simpson 2005, } \\
\text { Simpson } 2006\end{array}$ & $\begin{array}{l}\text { EPR } \\
\text { Clomipramina } \\
\text { EPR+Clomipramina }\end{array}$ & $\begin{array}{l}18 \\
11 \\
15\end{array}$ & 12 semanas \\
\hline O'Connor 2005 & $\begin{array}{l}\text { EPR } \\
\text { IBA } \\
\text { CAM }\end{array}$ & $\begin{array}{l}12 \\
16 \\
16\end{array}$ & 20 semanas \\
\hline Whittal 2005 & $\begin{array}{l}\text { EPR } \\
\text { TCog }\end{array}$ & $\begin{array}{l}37 \\
34\end{array}$ & 12 semanas \\
\hline Tenneij 2005 & $\begin{array}{l}\text { Paroxetina+ } \\
\text { Venlafaxina } \\
\text { Paroxetina+ } \\
\text { Venlafaxina+EPR }\end{array}$ & $\begin{array}{l}49 \\
47\end{array}$ & 24 semanas \\
\hline
\end{tabular}

\begin{tabular}{|c|c|c|c|}
\hline Estudo & Tratamento & $\mathbf{n}$ & $\begin{array}{l}\text { Duração do } \\
\text { tratamento }\end{array}$ \\
\hline Nakatani 2005 & $\begin{array}{l}\text { EPR } \\
\text { Fluvoxamina } \\
\text { Placebo }\end{array}$ & $\begin{array}{c}11 \\
11 \\
9\end{array}$ & 12 semanas \\
\hline Asbahr 2005 & $\begin{array}{l}\text { EPR+TCog grupo } \\
\text { Sertralina }\end{array}$ & $\begin{array}{l}20 \\
20\end{array}$ & 12 semanas \\
\hline Althaus 2005 & $\begin{array}{l}\text { T. TOC grupo+ } \\
\text { EPR+TCog } \\
T \text {. não-especifica } \\
\text { grupo+T. individual }\end{array}$ & $\begin{array}{l}14 \\
16\end{array}$ & 94,3 dias (média) \\
\hline Lovell 2006 & $\begin{array}{l}\text { EPR terapeuta } \\
\text { EPR telefone }\end{array}$ & $\begin{array}{l}36 \\
36\end{array}$ & $\begin{array}{l}10 \text { semanas } \\
9 \text { semanas }\end{array}$ \\
\hline Sousa 2006 & $\begin{array}{l}\text { EPR+TCog } \\
\text { Sertralina }\end{array}$ & $\begin{array}{l}28 \\
28\end{array}$ & 12 semanas \\
\hline O'Connor 2006 & $\begin{array}{l}\text { Fluvoxamina } \\
+ \text { +EPR+TCog } \\
\text { Placebo+EPR+TCog } \\
\text { EPR+TCog em } \\
\text { pacientes com IRS } \\
\text { EPR+TCog em } \\
\text { pacientes sem } \\
\text { medicação }\end{array}$ & $\begin{array}{l}10 \\
12 \\
11 \\
10\end{array}$ & $\begin{array}{c}5 \text { meses } \\
\text { (aproximadamente) }\end{array}$ \\
\hline Vogel 2006 & $\begin{array}{l}\text { EPR+ TCog } \\
\text { EPR+ relaxamento }\end{array}$ & $\begin{array}{l}16 \\
19\end{array}$ & 6 semanas \\
\hline Simons 2006 & $\begin{array}{l}\text { EPR } \\
\text { TMC }\end{array}$ & $\begin{array}{l}5 \\
5\end{array}$ & 20 semanas \\
\hline
\end{tabular}

EPR = Exposição e Prevenção de Resposta; TCog = Terapia Cognitiva; Exi = Exposição Imaginária; IBA = Inference-Based Approach; $C A M=$ Cognitive Appraisal Model; TMC =Terapia Metacognitiva; T. TOC grupo=Terapia especifica para TOC em grupo; $T$. não-especifica grupo = Terapia nãoespecifica em grupo; $T$. individual = Terapia individual $; I R S=$ Inibidores de recaptação da serotonina

3. EPR guiada por computador e EPR guiada por telefone

Um estudo com 218 pacientes com TOC comparou a eficácia de dez semanas de EPR guiada 1) por computador; 2) por um terapeuta comportamental; ou 3) por um guia de relaxamento sistemático. Para o TOC, a EPR guiada por um computador foi eficaz, embora aquela guiada por um terapeuta tenha se mostrado significativamente superior às demais intervenções. 0 relaxamento sistemático não foi eficaz. Estes resultados sugerem, portanto, que a EPR guiada por computador pode ser útil como um primeiro passo para tratar pacientes com TOC quando um terapeuta não for acessível. ${ }^{15}$

De maneira semelhante, um recente estudo de 2006 , com 72 pacientes com TOC, comparou a eficácia de dez sessões de EPR guiada por telefone com dez sessões guiadas por um terapeuta e mostrou que a taxa de resposta da EPR guiada por telefone foi equivalente ao tratamento realizado diretamente com o terapeuta e que altos níveis de satisfação (segundo o "questionário de satisfação do cliente" aplicado) foram alcançados por ambos os grupos. ${ }^{16}$

\section{EPR vs. TCog}

Um estudo recente realizado por O'Connor et al. comparou a eficácia da EPR com a TCog utilizando duas abordagens cognitivas diferentes: uma baseada em inferências primárias [inference-based approach (IBA)] e outra baseada no modelo cognitivo de avaliação de inferências secundárias [cognitive appraisal model (CAM)]. Na IBA, a dúvida 
Tabela 2 - Meta-análises publicadas entre 1995 e 2006

\begin{tabular}{lcc}
\hline \multicolumn{1}{c}{ Estudo } & Tratamento & $\mathbf{n}^{\mathbf{0}}$ de estudos analisados \\
\hline Abramowitz 1997 & $\begin{array}{c}\text { farmacológico } \\
\text { psicoterápico }\end{array}$ & 32 \\
Kobak 1998 & psicoterápico & 77 \\
Abramowitz 1998 & $\begin{array}{c}\text { psicoterápico } \\
\text { farmacológico }\end{array}$ & 10 \\
Ruhmland 2001 & psicoterápico & 32 \\
Foa 2002 & farmacológico & 9 \\
& farmacológico & 37 \\
Eddy 2004 & psicoterápico & 8 \\
Fisher 2005 & psicoterápico & 18 \\
Abramowitz 2005 & psicoterápico & \\
\hline
\end{tabular}

inicial (e.g. "Talvez eu esteja contaminado") é considerada uma inferência primária. Aqui, o tratamento foca no padrão de racionalidade que mantém a dúvida, ou seja, no fato de que a dúvida inicial é mantida por um processo racional idiossincrásico que leva a outras dúvidas (e.g. "Eu posso ter contaminado a minha família") e que, por sua vez, pode levar a avaliações negativas (e.g. "É horrível ter esses pensamentos"; "Estou fora de controle"). Já no CAM, são estas dúvidas e avaliações negativas secundárias à dúvida inicial que são discutidas com o paciente a partir de técnicas cognitivas. Neste estudo, 54 pacientes com TOC foram randomizados nesses três grupos de tratamento (EPR, IBA ou CAM). Após 20 semanas de tratamento, os três grupos revelaram eficácias semeIhantes, mostrando uma redução significativa nos escores do Yale-Brown Obsessive Compulsive Scale (Y-BOCS) e do Inventário Pádua.

Em um estudo controlado realizado com 71 pacientes com TOC submetidos à TCog, nos moldes de Beck ${ }^{7}$ e Salkovskis, ${ }^{9}$ a EPR demonstrou que ambas as terapias resultaram em decréscimos estatisticamente significativos na gravidade dos sintomas do TOC. No entanto, naqueles pacientes tratados com TCog, foram observadas reduções estatisticamente significativas em todas as medidas de eficácia analisadas, tanto aquelas diretamente relacionadas ao TOC (sintomas obsessivo-compulsivos) quanto aquelas relacionadas às psicopatologias associadas. Embora a melhora observada no grupo tratado com EPR não tenha sido tão ampla quanto aquela observada no grupo tratado com TCog, um número significativamente menor de pacientes do primeiro grupo apresentou recaída. ${ }^{18} \mathrm{Em}$ outro estudo envolvendo TCog e EPR com 65 pacientes com TOC, ambos os tratamentos mostraram-se eficazes para o TOC, sem diferença significativa entre os grupos. No entanto, o póstratamento da TCog indicou maiores efeitos na depressão do que a EPR. ${ }^{19}$ Resultados semelhantes foram observados em um estudo recente que comparou a eficácia da EPR com a TCog no pós-tratamento e no follow-up após três meses, no qual não foi encontrada diferença significativa nos escores do Y-BOCS dos pacientes que receberam TCog ou EPR. Apesar de uma maior porcentagem de recuperação entre os pacientes tratados com TCog no pós-tratamento (67\%) e follow-up (76\%) comparados aos tratados com EPR (59\% no pós-tratamento e 58\% no follow-up), esta diferença não foi significativa. ${ }^{20}$

\section{EPR e/ou TCog vs. medicação}

Recentemente, dois estudos controlados, realizados com 122 pacientes com TOC, compararam os efeitos da clomipramina (CMI) e da EPR após 12 semanas de tratamento ${ }^{21}$ e 12 sema- nas após sua descontinuidade. ${ }^{22}$ Os resultados mostraram que os respondedores à EPR (com ou sem o uso concomitante de CMI) obtiveram uma taxa significativamente menor de recaídas e maior tempo para recaída após o término do tratamento quando comparados aos pacientes respondedores a $\mathrm{CMI}$ isoladamente. A partir dessa mesma amostra, uma análise posterior foi realizada utilizando-se diferentes definições de recaída baseadas em critérios usados nos principais estudos com TOC (e.g. retorno a gravidade dos sintomas do pré-tratamento ou algum grau de piora da gravidade dos sintomas em relação ao pós-tratamento), confirmando que a EPR (com ou sem o uso de $\mathrm{CMI}$ ) pode produzir maior durabilidade dos ganhos em curto-prazo mesmo após a descontinuidade do tratamento do que a monoterapia com CMI. No entanto, alguns critérios de recaída, como "ter piora mínima", não foram significativamente diferentes entre os grupos. ${ }^{23}$ Mais recentemente, partindo desta mesma amostra, outra análise revelou que a EPR (com ou sem o uso de CMI) pode trazer resultado superior no tratamento de pacientes com TOC sem a comorbidade de depressão quando comparada com a CMI isoladamente. ${ }^{24}$

Tenneij et al. realizaram um estudo com 96 pacientes com TOC respondedores a um tratamento farmacológico por três meses, com o objetivo de examinar o quanto a adição de terapia comportamental (EPR) pode aumentar a resposta ao tratamento. Pacientes que já haviam recebido tratamento com paroxetina e venlafaxina foram randomizados em dois grupos: um recebeu tratamento adicional de EPR e o outro continuou com o tratamento farmacológico apenas. Os pacientes que receberam tratamento adicional de EPR mostraram maior taxa de melhora nos sintomas obsessivo-compulsivos (mudança no escore do $\mathrm{Y}$-BOCS $=-3,9$ para os que completaram o tratamento) em comparação aos pacientes que continuaram com a medicação apenas (mudança no escore do Y-BOCS $=+$ 3,9 para os que completaram o tratamento). ${ }^{25}$

Outros estudos encontraram resultados controversos sobre a eficácia da potencialização de terapias de base cognitivocomportamental por meio do uso de inibidores da recaptação da serotonina (e.g. fluvoxamina). Por exemplo, enquanto Hohagen et al. ${ }^{26}$ observaram que a EPR associada à fluvoxamina foi superior à EPR associada ao placebo, van Balkom et al. ${ }^{27}$ demonstraram que a EPR, a TCog e a fluvoxamina combinada com a EPR ou com a TCog resultaram em uma mesma magnitude de resposta no pós-teste do tratamento, não detectando qualquer efeito adicional da fluvoxamina sobre a EPR.

Um recente estudo também comparou a eficácia da fluvoxamina e da EPR para o tratamento de pacientes com TOC. Trinta e um pacientes foram randomizados em três grupos [i.e. EPR, fluvoxamina e placebo (grupo controle)], tratados por 12 semanas. Os pacientes tratados nos grupos de EPR e fluvoxamina apresentaram uma melhora significativamente maior do que aquela observada no grupo controle em relação ao escore total do Y-BOCS; o grupo tratado com EPR mostrou uma redução no escore total do Y-BOCS ao final do tratamento significativamente maior do que aquela observada no grupo tratado com fluvoxamina. ${ }^{28}$

Um estudo realizado em 2006 comparou a TCog aliada à EPR em grupo com o tratamento apenas com sertralina, revelando que ambos os tratamentos mostraram-se eficazes. No entanto, a taxa de remissão de sintomas, a redução da intensidade das compulsões e a porcentagem de pacientes que obtiveram remissão total foram significativamente maiores no grupo tratado com TCog +EPR em grupo. ${ }^{29}$ 
Outro estudo também comparou a eficácia da TCog aliada à EPR com o tratamento medicamentoso e mostrou que todos os tratamentos ativos resultaram em melhora, não havendo diferença significativa na resposta ao tratamento entre o grupo que recebeu EPR + TCog isoladamente e os grupos que anteriormente já haviam recebido medicação (fluvoxamina ou outros IRS) ou placebo. ${ }^{30}$

6. Predominância de pensamentos obsessivos no TOC

Um ensaio clínico randomizado mostrou que as obsessões podem melhorar com terapias que combinam abordagens comportamentais e cognitivas. Vinte e nove pacientes com TOC com predominância de pensamentos obsessivos submetidos à EPR e à reestruturação cognitiva obtiveram melhora significativa comparados à lista de espera. Após receber o tratamento, o grupo de espera também apresentou melhora clinicamente significativa. Os ganhos com a EPR e a reestruturação cognitiva foram mantidos nos seis meses subseqüentes. ${ }^{31}$

\section{Preditores de resposta}

Um estudo com 46 pacientes com TOC demonstrou que 0 melhor preditor de resposta ao final do tratamento e no followup foi a compreensão, ainda na primeira semana de tratamento, do modo de realização dos exercícios de EPR na tarefa de casa. Outro preditor de boa resposta encontrado no followup, embora menos consistente, foi a redução de ansiedade entre as semanas zero e quatro de tratamento. ${ }^{32}$

Outro estudo comparou a EPR guiada por computador ou por um terapeuta e o relaxamento, com o objetivo de identificar quais dimensões de sintomas de TOC estão associadas à aderência e à resposta à EPR. Pacientes com sintomas de colecionismo apresentaram maior desistência no início do tratamento e tenderam a apresentar uma melhora menos significativa dos sintomas. O fator "obsessão sexual/religiosa" também se mostrou um preditor de resposta desfavorável a EPR. ${ }^{33}$

Segundo análise de um estudo controlado realizado com 99 pacientes com TOC submetidos a 16 sessões em quatro condições de tratamento (TCog; EPR; TCog + fluvoxamina; EPR + fluvoxamina), a baixa motivação para terapia e a maior gravidade do transtorno foram considerados preditores de resposta desfavoráveis ao tratamento. Neste estudo, dos 45 pacientes classificados como não-respondedores no pós-tratamento, 17 tornaram-se respondedores no follow-up. Não foi encontrado nenhum preditor de resposta especificamente relacionado aos tratamentos adotados (TCog, EPR e tratamentos combinados). ${ }^{34}$

Os resultados de um estudo controlado realizado com 52 pacientes com TOC sugerem que pacientes com TOC e transtorno de personalidade (TP) concomitante não devem ser excluídos de tratamentos cognitivos ou comportamentais para o TOC. Os pacientes com TOC e TP, segundo a DSM-III-R, quando comparados aos pacientes com TOC sem TP, obtiveram benefícios equivalentes até pelo menos seis meses após o término da terapia. ${ }^{35}$

Um estudo recente demonstrou, a partir de uma análise de regressão, que uma aliança terapêutica foi um preditor de resposta significativo (segundo o Y-BOCS) e que a "expectativa em relação ao tratamento" e a "alta motivação para mudança" não se revelaram como variáveis significativas ao final do tratamento para o TOC. ${ }^{36}$

\section{TOC em crianças e adolescentes}

Em um estudo controlado, pacientes com TOC com idades entre 10 e 17 anos foram incluídos em dois grupos: um tratado apenas com fluvoxamina e o outro tratado com fluvoxamina e EPR. Os pacientes que receberam o tratamento combinado apresentaram melhora significativamente maior que os pacientes que receberam apenas a medicação. 0 mesmo foi observado no follow-up após dois anos do término do tratamento, sugerindo que o uso combinado de medicação e EPR é mais eficaz no tratamento do TOC em crianças e adolescentes. ${ }^{37}$

Outro estudo realizado com 112 pacientes com TOC, com idades entre 7 e 17 anos, também apontou para uma melhora significativamente superior no grupo de pacientes que recebeu tratamento combinado de sertralina e EPR associada à TCog quando comparado aos grupos que receberam EPR associada à TCog, sertralina ou placebo isoladamente. De acordo com a Child Yale-Brown Obsessive Compulsive Scale (CYBOCS), os pacientes nos grupos em monoterapia com EPR combinada à TCog e sertralina obtiveram resultados semeIhantes, ambos estatisticamente superiores ao placebo. ${ }^{38}$

Recentemente, um estudo realizado com 40 pacientes com TOC, com idades entre 9 e 17 anos, comparou a eficácia da sertralina em relação à EPR associada à TCog em grupo ambos por 12 semanas. Os dois tratamentos mostraram meIhora significativa nos sintomas obsessivo-compulsivos de acordo com o CY-BOCS. Após nove meses do término do tratamento, os pacientes tratados no grupo com EPR combinada à TCog apresentaram uma taxa de recaída significativamente menor do que aqueles tratados com sertralina somente. ${ }^{39}$

Outro estudo envolvendo terapia em grupo contou com a participação de 77 pacientes com TOC, com idade entre 7 e 17 anos, submetidos a 14 sessões de EPR associada à TCog familiar em grupo, EPR associada à TCog familiar individual ou quatro a seis semanas na lista de espera (grupo controle). Ambos os tratamentos com EPR associada à TCog familiar (individual e em grupo) produziram redução significativa nas taxas diagnósticas de TOC e na gravidade dos sintomas obsessivo-compulsivos comparados ao grupo controle. A intervenção em grupo mostrou ser tão efetiva em reduzir sintomas do TOC em crianças e adolescentes quanto a individual. Os ganhos foram mantidos após seis meses do término do tratamento. ${ }^{40}$

Simons et al. conduziram um estudo com dez crianças e adolescentes com TOC, com idades entre 8 e 17 anos, submetidos a 20 sessões de EPR ou de terapia metacognitiva (TMC). A principal diferença entre os dois protocolos de tratamento foi a interpretação das obsessões e o mecanismo dos exercícios propostos: na TMC, a EPR pode ser utilizada como um experimento comportamental, objetivando uma mudança nas avaliações metacognitivas disfuncionais, diferente da EPR propriamente dita, onde os pacientes são encorajados a realizar os exercícios repetidamente orientados pelo conceito da habituação. Por exemplo, em um paciente cujas obsessões envolvem medo de morrer apenas por pensar na morte, a intervenção metacognitiva incluiria a "aceitação" de tais pensamentos seguida por experimentos como tentar ganhar na loteria apenas com o poder da mente ou esforçar-se para cair no sono durante o dia. Neste estudo, todos os pacientes revelaram um decréscimo na gravidade dos sintomas obsessivo-compulsivos e, após três meses e dois anos do término do tratamento, os ganhos foram mantidos para todo o grupo estudado. ${ }^{41}$

\section{Terapia em grupo}

A eficácia de um programa de tratamento específico para TOC foi investigada no trabalho de Althaus et al. com 30 pacientes internados. Neste estudo, o grupo experimental 
recebeu tratamento em grupo específico para o TOC em associação à terapia de EPR aliada à TCog individual e o grupo controle recebeu terapia não-específica em grupo juntamente com terapia individual. No pós-tratamento, ambos os grupos apresentaram melhora significativa, não apresentando diferenças significativas nas medidas clínicas, dos terapeutas e na auto-avaliação. Porém, houve um número significativamente maior de respondedores (considerando respondedores os pacientes com melhora maior que 50\% no Y-BOCS) no grupo experimental. ${ }^{42}$

Um estudo que contou com 76 pacientes com TOC, tratados em grupos por dois terapeutas, durante 12 semanas, comparou os efeitos da TCog e da EPR em grupo para o tratamento do TOC. Ambos os tratamentos obtiveram resultados superiores à lista de espera (grupo controle). No entanto, ao final do tratamento, a EPR mostrou-se marginalmente mais eficaz que a TCog. Além disso, uma proporção significativamente maior de pacientes dentre aqueles tratados com EPR foi considerada "melhor" do que aqueles tratados com TCog após três meses. ${ }^{43}$

Neste estudo, McLean et al. sugeriram que a EPR pode ser mais adequada para o tratamento em grupo do TOC por dois motivos. Em primeiro lugar, a pressão exercida pelos participantes, fator próprio do tratamento em grupo, poderia trazer maiores ganhos em relação à compreensão da EPR. Segundo, o treinamento de terapeutas em EPR seria substancialmente mais fácil do que nos métodos da TCog. No entanto, um outro estudo controlado realizado com pacientes com TOC mostrou que a EPR combinada à TCog em grupo reduz os sintomas do TOC e as idéias supervalorizadas, e melhora a qualidade de vida dos pacientes de uma forma estável, possibilitando um menor custo no tratamento de um maior número de pacientes com TOC. ${ }^{6}$ Neste estudo, 23 pacientes com TOC receberam 12 sessões semanais de duas horas de EPR aliada à TCog em grupo e foram comparados a 24 pacientes com TOC que aguardaram na lista de espera (grupo controle). O percentual de pacientes que obtiveram melhora foi de $69,6 \%$ entre os pacientes tratados e de $4,2 \%$ entre os controles ( $p<0,001$ ). Três meses após o término do grupo, uma redução significativa adicional nos sintomas do TOC foi ainda constatada. ${ }^{6}$

\section{Meta-análises}

Os 32 estudos controlados envolvendo tratamento para o TOC, publicados entre 1975 e 1995, analisados na revisão quantitativa realizada por Abramowitz em 1997,44 mostraram que a EPR foi altamente eficaz na redução dos sintomas do TOC e que as abordagens cognitivas também foram, ao menos, tão efetivas quanto a EPR. Fisher et al. encontraram resultados semelhantes ao analisar oito estudos controlados envolvendo somente tratamentos psicoterápicos - i.e. neste estudo, a EPR foi o mais efetivo tratamento psicoterápico do TOC. ${ }^{45}$ Em 1998, Abramowitz reavaliou a eficácia da EPR no tratamento do TOC; porém, nesta revisão, incluiu apenas estudos que utilizaram o Maudsley Obsessional-Compulsive Invetory (MOCl) como medida de resposta. Ao todo, dez estudos, publicados entre 1983 e 1995, foram avaliados e os resultados confirmaram a eficácia da EPR em reduzir os sintomas obsessivo-compulsivos, principalmente rituais de checagem e lavagem. Embora os pacientes pesquisados tenham apresentado um comportamento mais próximo da população geral do que da população acometida por TOC ao término do tratamento, o autor considera que a EPR não pa- receu curar estes pacientes, visto que alguns deles ainda se mantiveram distintos da população geral. ${ }^{46}$

Kobak et al. analisaram 77 estudos com o objetivo de avaliar quantitativamente a eficácia de cinco inibidores de recaptação de serotonina (IRS) [i.e.clomipramina, fluoxetina, sertralina, paroxetina e fluvoxamina] e da terapia comportamental (EPR). O tamanho de efeito da EPR foi significativamente maior que o dos IRS como um todo, mas a diferença não foi significativa quando variáveis metodológicas foram controladas. O tamanho do efeito da intervenção combinada de EPR e IRS não foi significativamente maior que o tamanho do efeito da EPR isolada. ${ }^{47}$

Um estudo alemão, de 2001, comparou a eficácia de diferentes tipos de tratamento psicoterápicos para o TOC e para fobia específica, partindo da análise de 32 estudos no total. No tratamento do TOC, a terapia cognitivo-comportamental (TCog aliada à EPR) apresentou o mais alto tamanho de efeito $(1,84)$ para os principais sintomas. O tamanho de efeito da EPR foi menor $(1,44)$, mas não houve diferença significativa quando este índice foi comparado ao tamanho de efeito da TCog aliada à EPR. Os ganhos obtidos com ambos os tratamentos foram mantidos após dois anos da intervenção. ${ }^{48}$

Outra meta-análise investigou a eficácia do tratamento psicoterápico (EPR, TCog e EPR aliada à TCog) e farmacológico (e.g. IRS, ansiolíticos, tricíclicos) avaliando apenas estudos controlados publicados entre 1980 e 2001. Os 15 estudos envolvendo psicoterapia e os 22 envolvendo tratamento medicamentoso replicaram as conclusões antes apontadas em outras revisões quantitativas que avaliaram a eficácia da psicoterapia no tratamento do TOC. Ambos os tipos de tratamentos produziram diminuição dos sintomas obsessivo-compulsivos, o que pôde ser observado nos tamanhos de efeito entre pré e pós-tratamento, na substancial porcentagem de pacientes que melhoraram com o tratamento e na considerável diminuição dos sintomas pré e pós-tratamento. ${ }^{49}$

Com o objetivo de avaliar as vantagens e desvantagens da combinação dos tratamentos farmacológico e psicoterápico de abordagem cognitivo-comportamental para o tratamento de transtornos de ansiedade, Foa et al. conduziram uma metaanálise na qual quatro estudos controlados com pacientes com TOC, entre estudos com pacientes com outros transtornos de ansiedade, foram analisados. O tamanho de efeito e a porcentagem de respondedores não foram significativamente diferentes entre os indivíduos tratados com combinações de medicação e psicoterapia ou psicoterapia isoladamente, sejam eles pacientes com TOC, fobia social ou transtorno de ansiedade generalizada. No entanto, a diferença nos protocolos de tratamento psicoterápicos, os desenhos de tratamentos que utilizaram simultaneamente a medicação e a psicoterapia, o pequeno número de estudos controlados incluídos, entre outras limitações, dificultaram a análise do tamanho de efeito e da porcentagem de respondentes no pós-tratamento e no follow-up, não sendo possível demonstrar de forma clara as vantagens e desvantagens do tratamento combinado. ${ }^{50}$

Em 2005, Abramowitz et al. conduziram a primeira metaanálise envolvendo o tratamento psicoterápico do TOC infantil. Dezoito estudos, publicados entre 1983 e 2004, foram examinados e os resultados mostraram que terapias de base cognitivo-comportamental envolvendo EPR e IRS (e.g. clomipramina e fluvoxamina) são eficazes em reduzir os sintomas obsessivo-compulsivos. As terapias de base cognitivo-comportamental produziram maiores tamanhos de 
efeito do que o tratamento medicamentoso, embora muitos estudos analisados tenham incluído metodologias diferentes e não controladas. ${ }^{51}$

\section{Discussão}

Os estudos controlados realizados na última década confirmam a eficácia da EPR no tratamento do TOC. Embora a TCog também tenha apresentado resultados favoráveis, mais estudos incluindo análise de recaída são necessários. Os ensaios controlados realizados com crianças mostram que o tratamento combinado de psicoterapia de base cognitivo comportamental associado à medicação anti-obsessiva é mais eficaz que o tratamento exclusivamente medicamentoso.

Os estudos realizados com terapia em grupo revelaram resultados favoráveis ao tratamento do TOC, o que possibilita um menor custo no tratamento de um maior número de pacientes. No entanto, estudos que avaliam os fatores potenciais que modificam a dinâmica do tratamento em grupo ainda são necessários (e.g. estudos que envolvam subgrupos de pacientes com TOC com predomínio de uma determinada dimensão de sintomas e seus respectivos preditores de resposta terapêutica).

A combinação de EPR e TCog também se mostrou eficaz na redução dos sintomas obsessivo-compulsivos, das idéias supervalorizadas e na melhora da qualidade de vida dos pacientes com TOC. A associação de TCog e EPR é uma estratégia que tem como objetivo aumentar a aderência e a resposta ao tratamento. No entanto, novas alternativas de tratamento precisam ser desenvolvidas em favor dos pacientes refratários e de pacientes com comorbidades, pois os estudos apresentados somente incluem pacientes sem determinados tipos de comorbidades, incluindo comorbidades graves (e.g. depressão maior atual; psicoses). Um estudo recente mostrou que apenas três horas de workshop sobre TCog e EPR podem resultar em diminuição significativa no número de sintomas obsessivo-compulsivos e de crenças do tipo fusão do pensamento e da ação em indivíduos com sintomas obsessivos-compulsivos subclínicos após cinco meses. ${ }^{52}$

Através da análise dos artigos controlados apresentados, percebemos que não há na literatura um consenso a respeito de uma definição sobre critérios de recaída e tempo ideal de acompanhamento dos pacientes após o término do tratamento (follow-up) que permita analisar adequadamente os ganhos e a eficácia dos diferentes tratamentos (TCog; EPR; TCog aliada à EPR e medicação). Apenas dois estudos (baseados na mesma amostra de pacientes) dentre os 33 ensaios clínicos randomizados identificados apresentam uma análise detalhada das taxas de recaída e remissão de sintomas partindo de diferentes definições. Em relação ao follow-up, 18 estudos dentre os 33 apresentam de forma clara que houve um acompanhamento pós-tratamento, sendo a maioria dos seguimentos realizados somente três meses após o término do tratamento. Portanto, faz-se necessário que um critério único de recaída seja estabelecido e que mais estudos de longo prazo sejam realizados. Um bom exemplo deste tipo de estudo mostrou que um número significativamente maior de pacientes tratados com fluvoxamina combinada a EPR ou TCog ainda fazia uso de antidepressivos cinco anos após o término do tratamento quando comparados aos pacientes tratados somente com TCog. ${ }^{53}$ De maneira semelhante, Rufer et al. acompanharam 30 de 37 pacientes com TOC seis a oito anos após o término do tratamento de base cognitivocomportamental (EPR+TCog) em combinação com fluvoxamina ou placebo num estudo controlado. ${ }^{54}$ Este estudo mostrou que os ganhos significativos observados comparando o pré e o póstratamento (redução de $41 \%$ no Y-BOCS) mantiveram-se no follow-up (45\%) e que a taxa de respondedores, definida como redução $\geqslant 35 \%$ no Y-BOCS, foi de $67 \%$ no pós-tratamento e $60 \%$ no follow-up. ${ }^{54}$ Somente a partir desses estudos será possível esclarecer se os ganhos com o tratamento persistem em longo prazo.

Uma revisão sistemática qualitativa como a nossa possui algumas limitações metodológicas intrínsecas que só podem ser solucionadas através de avaliações quantitativas (e.g gráficos de meta-análise ou blobbograms). ${ }^{55}$ Por exemplo, parâmetros como razões de chances, intervalos de confiança e peso de cada ensaio clínico randomizado não foram descritos, o que compromete uma avaliação mais precisa da eficácia de cada abordagem terapêutica por nós revisada.

\section{Conclusão}

Nos últimos dez anos, novos estudos confirmam que a EPR e a TCog são eficazes no tratamento do TOC de crianças, adolescentes e adultos. Em adultos, o uso concomitante de medicação anti-obsessiva (e.g. IRS) e EPR ou TCog não tem sido acompanhado de um efeito adicional na resposta ao tratamento. Em crianças e adolescentes com TOC, no entanto, a maioria dos estudos sugere que existe uma superioridade do uso combinado de medicação e EPR em relação aos dois tratamentos isoladamente. A EPR associada à TCog resultou em benefícios significativos em pacientes com predominância de pensamentos obsessivos e sua modalidade em grupo também produziu redução significativa dos sintomas obsessivos e compulsivos.

Embora avanços importantes tenham sido obtidos nos últimos anos (e.g. aumento no número de estudos incluindo TCog, IRS e placebo), ainda existe uma série de lacunas no conhecimento atual sobre o papel da EPR e da TCog no tratamento do TOC. Por exemplo, ainda não está claro em quais momentos, em que casos específicos e como as terapias de base cognitivocomportamental do TOC podem otimizar os resultados do tratamento farmacológico prévio. ${ }^{25}$ Pesquisas adicionais sobre diferentes modalidades de tratamento em grupo e sobre o uso combinado de medicação e psicoterapia também precisam ser desenvolvidas. Além disso, estudos de análise de recaída, com pacientes refratários ou diferentes comorbidades devem envolver períodos de follow-up mais longos e tornar mais explícitas as potenciais causas do fracasso do tratamento. ${ }^{56}$

\section{Referências}

1. Fontenelle LF, Mendlowicz MV, Versiani M. The descriptive epidemiology of obsessive-compulsive disorder. Prog Neuropsychopharmacol Biol Psychiatry. 2006;30(3):327-37.

2. Abramowitz JS, Whiteside SP, Deacon BJ. The effectiveness of treatment for pediatric obsessive-compulsive disorder: a metaanalysis. Behav Therapy. 2005;36(1):55-63.

3. Huppert JD, Schultz LT, Foa EB, Barlow DH, Davidson JR, Gorman JM, Shear MK, Simpson HB, Woods SW. Differential response to placebo among patients with social phobia, panic disorder, and obsessive-compulsive disorder. Am J Psychiatry. 2004;161(8): 1485-7.

4. March JS, Mulle K. OCD in children and adolescents: a cognitivebehavioral treatment manual. New York: The Guilford Press; 1998.

5. Marks I. Behaviour therapy for obsessive-compulsive disorder: a decade of progress. Can J Psychiatry. 1997;42(10):1021-7.

6. Volpato Cordioli A, Heldt E, Braga Bochi D, Margis R, Basso de Sousa M, Fonseca Tonello J, Gus Manfro G, Kapczinski F. Cognitivebehavioral group therapy in obsessive-compulsive disorder: a randomized clinical trial. Psychother Psychosom. 2003;72(4):211-6. 
7. Beck AT. Cognitive therapy and the emotional disorder. New York: International Universities Press; 1976.

8. Beck AT, Emery G, Greenberg R. Anxiety disorders and phobias. A cognitive perspective. New York: Basic Books; 1985.

9. Salkovskis PM. Obsessional-compulsive problems: a cognitivebehavioural analysis. Behav Res Ther. 1985;23(5):571-83.

10. Salkovskis PM, Kirk J. Obsessional disorders. In: Hawton K, Salkovskis PM, Kirk J, Clark D, editors. Cognitive behaviour therapy for psychiatric problems. A practical guide. Oxford: Oxford University Press; 1989. p. 128-68.

11. Marks IM. Fears, phobias and rituals. New York: Oxford University Press; 1987.

12. Lindsay M, Crino R, Andrews G. Controlled trial of exposure and response prevention in obsessive-compulsive disorder. $\mathrm{Br} \mathrm{J}$ Psychiatry. 1997;171:135-9.

13. de Araujo LA, Ito LM, Marks IM, Deale A. Does imagined exposure to the consequences of not ritualising enhance live exposure for OCD? A controlled study. I. Main outcome. Br J Psychiatry. $1995 ; 167(1): 65-70$.

14. Ito LM, Marks IM, de Araujo LA, Hemsley D. Does imagined exposure to the consequences of not ritualising enhance live exposure for OCD? A controlled study. II. Effect on behavioral versus subjective concordance of improvement. Br J Psychiatry. 1995;167(1):71-5.

15. Greist JH, Marks IM, Baer L, Kobak KA, Wenzel KW, Hirsch MJ, Mantle JM, Clary CM. Behavior therapy for obsessive-compulsive disorder guided by a computer or by a clinician compared with relaxation as a control. J Clin Psychiatry. 2002;63(2):138-45.

16. Lovell K, Cox D, Haddock G, Jones C, Raines D, Garvey R, Roberts C, Hadley S. Telephone administered cognitive behaviour therapy for treatment of obsessive compulsive disorder: randomised controlled non-inferiority trial. BMJ. 2006;333(7574):883-7.

17. O'Connor KP, Aardema F, Bouthillier D, Fournier S, Guay S, Robillard S, Pelissier MC, Landry P, Todorov C, Tremblay M, Pitre D. Evaluation of an inference-based approach to treating obsessive-compulsive disorder. Cogn Behav Ther. 2005;34(3):148-63.

18. van Oppen P, de Haan E, van Balkom AJ, Spinhoven P, Hoogduin K, van Dyck R. Cognitive therapy and exposure in vivo in the treatment of obsessive compulsive disorder. Behav Res Ther. 1995;33(4):379-90.

19. Cottraux J, Note I, Yao SN, Lafont S, Note B, Mollard E, Bouvard M, Sauteraud A, Bourgeois M, Dartigues JF. A randomized controlled trial of cognitive therapy versus intensive behavior therapy in obsessive-compulsive disorder. Psychother Psychosom. 2001;70(6):288-97.

20. Whittal ML, Thordarson DS, McLean PD. Treatment of obsessivecompulsive disorder: cognitive behavior therapy vs. exposure and response prevention. Behav Res Ther. 2005;43(12):1559-76.

21. Foa EB, Liebowitz MR, Kozak MJ, Davies S, Campeas R, Franklin ME, Huppert JD, Kjernisted K, Rowan V, Schmidt AB, Simpson HB, Tu X. Randomized, placebo-controlled trial of exposure and ritual prevention, clomipramine, and their combination in the treatment of obsessive-compulsive disorder. Am J Psychiatry. 2005;162(1):151-61

22. Simpson HB, Liebowitz MR, Foa EB, Kozak MJ, Schmidt AB, Rowan V, Petkova E, Kjernisted K, Huppert JD, Franklin ME, Davies SO, Campeas R. Post-treatment effects of exposure therapy and clomipramine in obsessive-compulsive disorder. Depress Anxiety. 2004;19(4):225-33.

23. Simpson HB, Franklin ME, Cheng J, Foa EB, Liebowitz MR. Standard criteria for relapse are needed in obsessive-compulsive disorder. Depress Anxiety. 2005;21(1):1-8.

24. Simpson HB, Huppert JD, Petkova E, Foa EB, Liebowitz MR. Response versus remission in obsessive-compulsive disorder. J Clin Psychiatry. 2006;67(2):269-76.

25. Tenneij NH, van Megen HJ, Denys DA, Westenberg HG. Behavior therapy augments response of patients with obsessive-compulsive disorder responding to drug treatment. J Clin Psychiatry. 2005;66(9):1169-75.

26. Hohagen F, Winkelmann G, Rasche-Ruchle H, Hand I, Konig A, Munchau N, Hiss H, Geiger-Kabisch C, Kappler C, Schramm P, Rey E, Aldenhoff J, Berger M. Combination of behaviour therapy with fluvoxamine in comparison with behaviour therapy and placebo. Results of a multicentre study. Br J Psychiatry Suppl. 1998;(35):71-8.
27. van Balkom AJ, de Haan E, van Oppen P, Spinhoven P, Hoogduin $K A$, van Dyck R. Cognitive and behavioral therapies alone versus in combination with fluvoxamine in the treatment of obsessive compulsive disorder. J Nerv Ment Dis. 1998;186(8):492-9.

28. Nakatani E, Nakagawa A, Nakao T, Yoshizato C, Nabeyama M, Kudo A, Isomura K, Kato N, Yoshioka K, Kawamoto M. A randomized controlled trial of Japanese patients with obsessive-compulsive disorder-effectiveness of behavior therapy and fluvoxamine. Psychother Psychosom. 2005;74(5):269-76.

29. Sousa MB, Isolan LR, Oliveira RR, Manfro GG, Cordioli AV. A randomized clinical trial of cognitive-behavioral group therapy and sertraline in the treatment of obsessive-compulsive disorder. J Clin Psychiatry. 2006;67(7):1133-9.

30. O'Connor KP, Aardema F, Robillard S, Guay S, Pelissier MC, Todorov C, Borgeat F, Leblanc V, Grenier S, Doucet P. Cognitive behaviour therapy and medication in the treatment of obsessive-compulsive disorder. Acta Psychiatr.Scand. 2006;113(5):408-19.

31. Freeston $\mathrm{MH}$, Ladouceur R, Gagnon F, Thibodeau N, Rheaume J, Letarte $\mathrm{H}$, Bujold A. Cognitive-behavioral treatment of obsessive thoughts: a controlled study. J Consult Clin Psychol. 1997;65(3):405-13.

32. de Araujo LA, Ito LM, Marks IM. Early compliance and other factors predicting outcome of exposure for obsessive-compulsive disorder. Br J Psychiatry. 1996;169(6):747-52.

33. Mataix-Cols D, Marks IM, Greist JH, Kobak KA, Baer L. Obsessivecompulsive symptom dimensions as predictors of compliance with and response to behaviour therapy: results from a controlled trial. Psychother Psychosom. 2002;71(5):255-62.

34. de Haan E, van Oppen P, van Balkom AJ, Spinhoven P, Hoogduin $K A$, van Dyck R. Prediction of outcome and early vs. late improvement in OCD patients treated with cognitive behaviour therapy and pharmacotherapy. Acta Psychiatr Scand. 1997;96(5):354-61.

35. Dreessen L, Hoekstra R, Arntz A. Personality disorders do not influence the results of cognitive and behavior therapy for obsessive compulsive disorder. J Anxiety Disord. 1997;11(5):503-21.

36. Vogel PA, Hansen B, Stiles TC, Gotestam KG. Treatment motivation, treatment expectancy, and helping alliance as predictors of outcome in cognitive behavioral treatment of OCD. J Behav Ther Exp Psychiatry. 2006;37(3):247-55.

37. Neziroglu F, Yaryura-Tobias JA, Walz J, McKay D. The effect of fluvoxamine and behavior therapy on children and adolescents with obsessive-compulsive disorder. J Child Adolesc Psychopharmacol. 2000;10(4):295-306.

38. Pediatric OCD Treatment Study (POTS) Team. Cognitive-behavior therapy, sertraline, and their combination for children and adolescents with obsessive-compulsive disorder: the Pediatric OCD Treatment Study (POTS) randomized controlled trial. JAMA. 2004;292(16):1969-76.

39. Asbahr FR, Castillo AR, Ito LM, Latorre MR, Moreira MN, LotufoNeto F. Group cognitive-behavioral therapy versus sertraline for the treatment of children and adolescents with obsessive-compulsive disorder. J Am Acad Child Adolesc Psychiatry. 2005;44(11):1128-36.

40. Barrett P, Healy-Farrell L, March JS. Cognitive-behavioral family treatment of childhood obsessive-compulsive disorder: a controlled trial. J Am Acad Child Adolesc Psychiatry. 2004;43(1):46-62.

41. S imons M, Schneider S, Herpertz-Dahlmann B. Metacognitive therapy versus exposure and response prevention for pediatric obsessive-compulsive disorder. A case series with randomized allocation. Psychother Psychosom. 2006;75(4):257-64.

42. Althaus D, Zaudig M, Hauke W, Roper G, Buttollo W. [The efficacy of an inpatient group therapy program for obsessive-compulsive disorder in addition to individual cognitive behavioral therapy and medication]. Verhaltenstherapie. 2000;10(1):16-23.

43. McLean PD, Whittal ML, Thordarson DS, Taylor S, Sochting I, Koch WJ, Paterson R, Anderson KW. Cognitive versus behavior therapy in the group treatment of obsessive-compulsive disorder. J Consult Clin Psychol. 2001;69(2):205-14.

44. Abramowitz JS. Effectiveness of psychological and pharmacological treatments for obsessive-compulsive disorder: a quantitative review. J Consult Clin Psychol. 1997 65(1):44-52.

45. Fisher PL, Wells A. How effective are cognitive and behavioral treatments for obsessive-compulsive disorder? A clinical significance analysis. Behav Res Ther. 2005;43(12):1543-58. 
46. Abramowitz JS. Does cognitive-behavioral therapy cure obsessivecompulsive disorder? A meta-analytic evaluation of clinical significance. Behav Therapy. 1998;29:339-55.

47. Kobak KA, Greist JH, Jefferson JW, Katzelnick DJ, Henk HJ. Behavioral versus pharmacological treatments of obsessive compulsive disorder: a meta-analysis. Psychopharmacology (Berl). 1998;136(3):205-16

48. Ruhmland M, Margraf J. Efficacy of psychological treatments for specific phobia and obsessive compulsive disorder. Verhaltenstherapie. 2001;11(1):14-26.

49. Eddy KT, Dutra L, Bradley R, Westen D. A multidimensional metaanalysis of psychotherapy and pharmacotherapy for obsessivecompulsive disorder. Clin Psychol Rev. 2004;24(8):1011-30.

50. Foa EB, Franklin ME, Moser J. Context in the clinic: how well do cognitive-behavioral therapies and medications work in combination? Biol Psychiatry. 2002;52(10):987-97.

51. Abramowitz JS, Whiteside SP, Deacon BJ. The effectiveness of treatment for pediatric obsessive-compulsive disorder: a metaanalysis. Behav Therapy. 2005;36(1):55-63.

52. Zucker BG, Craske MG, Blackmore MA, Nitz AA. Cognitive behavioral workshop for subclinical obsessions and compulsions. Behav Res Ther. 2006;44(2):289-304

53. van Oppen P, van Balkom AJ, de Haan E, van Dyck R. Cognitive therapy and exposure in vivo alone and in combination with fluvoxamine in obsessive-compulsive disorder: a 5-year follow-up. J Clin Psychiatry. 2005;66(11):1415-22.

54. Rufer M, Hand I, Alsleben H, Braatz A, Ortmann J, Katenkamp B, Fricke S, Peter H. Long-term course and outcome of obsessivecompulsive patients after cognitive-behavioral therapy in combination with either fluvoxamine or placebo: a 7-year follow-up of a randomized double-blind trial. Eur Arch Psychiatry Clin Neurosci. 2005;255(2):121-8 .

55. Gray GE. Psiquiatria baseada em evidências. Porto Alegre: Artmed; 2004.

56. Neziroglu F, Henricksen J, Yayura-Tobias J. Psychoterapy of obsessivecompulsive disorder and spectrum: established facts and advances, 1995-2005. Psychiatr Clin N Am. 2006;29(2):585-604. 